\title{
Erratum: Coherent synchrotron radiation instability in a bunch compressor [Phys. Rev. ST Accel. Beams 5, 064401 (2002)]
}

\author{
S. Heifets, G. Stupakov, and S. Krinsky
}

(Received 18 November 2002; published 27 December 2002)

DOI: 10.1103/PhysRevSTAB.5.129902

PACS numbers: 29.27.-a, 99.10.Cd

In the our original paper the sign of the coefficient $R_{56}$ used in numeric calculations was wrong. It has to be changed to be consistent with the sign convention assumed in the design of a bunch compressor. The sign of $R_{56}$ depicted in Fig. 1 has to be changed from negative to positive. The sign of the chirp $u$ related to the sign of the $R_{56}$ has to be changed as well: the chirp $u$ in the first paragraph of Sec. VI should be positive, $u=+39.83 \mathrm{~m}^{-1}$. These changes do not affect the formulas of the paper but slightly modify the numeric results. The results of corrected calculations are shown in Figs. 1 and 2 given below, which should replace Figs. 3 and 4 in the paper, respectively.

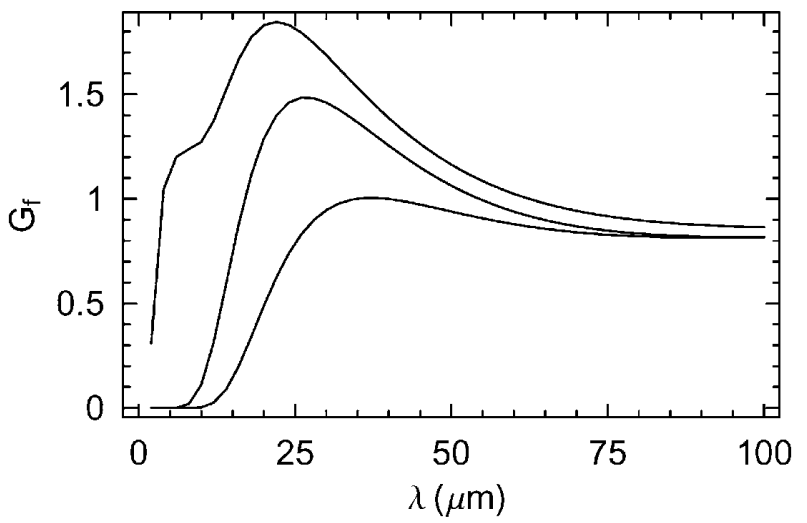

FIG. 1. Amplification factor $G_{f}$ as a function of wavelength $\lambda$ of the perturbation at the compressor entrance for various beam emittance and energy spread: (1) $\sigma_{p}=3.0 \times$ $10^{-5}, \epsilon=1 \mu \mathrm{m}$; (2) $\sigma_{p}=3.0 \times 10^{-5}, \epsilon=0$; (3) $\sigma_{p}=3.0 \times$ $10^{-6}, \epsilon=1 \mu \mathrm{m}$.

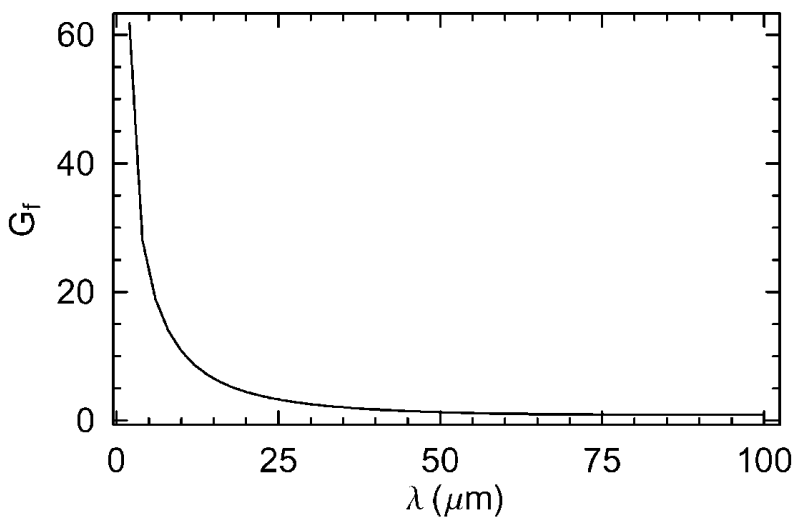

FIG. 2. Amplification factor $G_{f}$ as a function of wavelength for the zero beam emittance and $\sigma_{p}=3.0 \times 10^{-6}$. 